\title{
Precision Higgs coupling measurements at the LHC through ratios of production cross sections
}

\author{
Abdelhak Djouadi ${ }^{\mathrm{a}}$ \\ Laboratoire de Physique Théorique, U. Paris-Sud and CNRS, 91405 Orsay, France \\ Theory Unit, Department of Physics, CERN, 1211 Geneva 23, Switzerland
}

Received: 8 February 2013 / Revised: 26 March 2013 / Published online: 12 July 2013

(C) The Author(s) 2013. This article is published with open access at Springerlink.com

\begin{abstract}
Now that the Higgs particle has been observed by the ATLAS and CMS experiments at the LHC, the next endeavor would be to probe its fundamental properties and to measure its couplings to fermions and gauge bosons with the highest possible accuracy. However, the measurements will be limited by significant theoretical uncertainties that affect the production cross section in the main production channels as well as by experimental systematical errors. Following earlier work, we propose in this paper to consider ratios of Higgs production cross sections times decay branching ratios in which most of the theoretical uncertainties and some systematical errors, such as the ones due to the luminosity measurement and the Higgs decay branching fractions, cancel out. The couplings of the Higgs particle could be then probed in a way that will be mostly limited by the statistical accuracy achievable at the LHC and accuracies at the percent level are foreseen for some of the ratios at the end of the LHC run. At the theoretical level, these ratios are also interesting as they do not involve the ambiguities that affect the Higgs total decay width in new physics scenarios. To illustrate how these ratios can be used to determine the Higgs couplings, we perform a rough analysis of the recent ATLAS and CMS data which shows that there is presently no significant deviation from the Standard Model expectation.
\end{abstract}

\section{Introduction}

It is expected since a long time that the probing of the mechanism that triggers the breaking of the electroweak symmetry and generates the fundamental particle masses will be, at least, a two chapters story. The first one is the search and the observation of a spin-zero Higgs particle that will confirm the scenario of the Standard Model (SM) and most of its extensions, that is, a spontaneous symmetry breaking by

a e-mail: djouadi@th.u-psud.fr a scalar field that develops a non-zero vacuum expectation value [1-4]. This long chapter has just been closed by the ATLAS and CMS collaborations with the spectacular observation of a new boson with a mass of $\approx 125 \mathrm{GeV}$ [5-7] and with, apparently, the basic properties required by the symmetry breaking mechanism in the SM [8-20]. This crucial observation opens a second and equally important chapter: the precise determination of the Higgs boson profile and the unraveling of the mechanism itself. In particular, a precise measurement of the Higgs couplings to fermions and gauge bosons (as well as its self-coupling) will be mandatory to establish the exact nature of the symmetry breaking mechanism and, eventually, to pin down effects of new physics if additional ingredients beyond those of the SM are involved [21].

Fortunately, the Higgs particle was born under a very lucky star which will make this second chapter rather eventful and exciting. Indeed, the mass value $M_{H} \approx 125 \mathrm{GeV}$ allows to produce the Higgs particle at the LHC in many redundant channels and to detect it in a large variety of decay modes. This is illustrated in Fig. 1 where, in the left-hand side, the decay branching fractions of the SM Higgs boson are displayed for the narrow mass range $M_{H}=$ $120-130 \mathrm{GeV}$ and it can be seen that the decay modes into $b \bar{b}, \tau^{+} \tau^{-}, W W^{*}$ and $Z Z^{*}$ final states are significant; this is also the case for the rare but clean loop induced decays $H \rightarrow \gamma \gamma$ and eventually $H \rightarrow Z \gamma$, and even the very rare $H \rightarrow \mu^{+} \mu^{+}$channel, which should be accessible with enough data. In the right-hand side of the figure, shown are the production rates at the LHC of a $125 \mathrm{GeV}$ SM Higgs boson for various past, present, and foreseen center of mass energies. While the by far dominant gluon-gluon fusion mechanism $g g \rightarrow H$ has extremely large rates, the subleading channels, i.e. the vector boson fusion (VBF) $q q \rightarrow H q q$, the Higgs-strahlung (HV) $q \bar{q} \rightarrow H V$ with $V=W, Z$ and the top quark associated $p \bar{p} \rightarrow t \bar{t} H$ mechanisms, have cross sections which should allow a study of the Higgs particle 
Fig. 1 The branching ratios of the SM Higgs boson in the mass range $M_{H}=120-130 \mathrm{GeV}$ (left) and its production cross sections at the LHC for various c.m. energies (right)

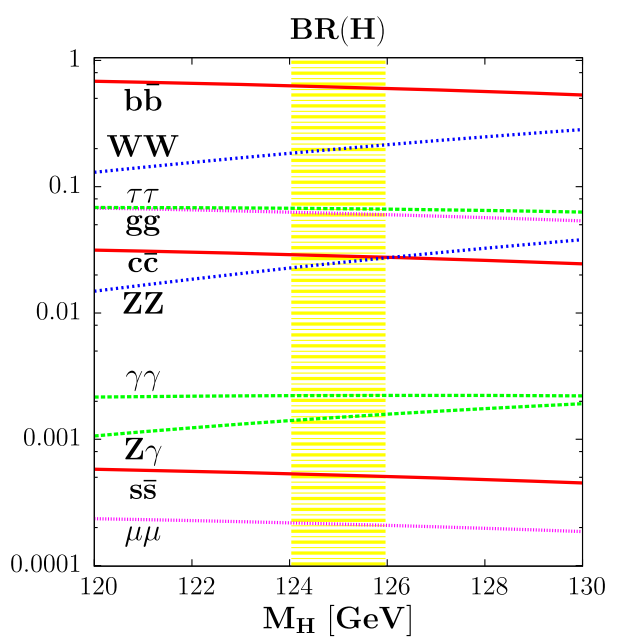

with $\sqrt{s} \gtrsim 14 \mathrm{TeV}$ once a large luminosity, $\gtrsim 100 \mathrm{fb}^{-1}$, has been collected.

The precision measurement chapter is already open as, a few days only after the Higgs discovery, a number of theoretical analyses have appeared to determine the Higgs couplings [8-20]. In fact the preamble has been written by the ATLAS and CMS collaborations themselves [6, 7] as they already quoted the values of the global signal strength modifier $\hat{\mu}$ which, with some approximation, can be identified with the Higgs cross section normalized to the SM expectation, when the various analyzed Higgs search channels are combined:

ATLAS: $\hat{\mu}=1.40 \pm 0.30$

CMS: $\hat{\mu}=0.87 \pm 0.23$

These first results already deliver two messages. An important first message is that the observed particle seems to approximately have the couplings to fermions and gauge bosons that are predicted by the SM, despite some small excesses and deficits that appear in some individual channels. A second message is that, already with the rather limited statistics at hand, the accuracy of the measurements in Eqs. (1)-(2) is reaching the $20 \%$ level for ATLAS and $\approx 25 \%$ for CMS. This is at the same time impressive and worrisome. Indeed, as illustrated in Fig. 1, the main Higgs production channel is the top and bottom quark loop mediated gluon-gluon fusion mechanism, $g g \rightarrow H$, and at $\sqrt{s}=7$ or $8 \mathrm{TeV}$, the three other mechanisms contribute at a level below $15 \%$ when their rates are added and before kinematical cuts are applied [22, 23]. Hence, the majority of signal events presently observed at the LHC, in particular in the main search channels $H \rightarrow \gamma \gamma, H \rightarrow Z Z^{*} \rightarrow 4 \ell^{ \pm}$, $H \rightarrow W W^{*} \rightarrow \ell^{+} \nu \ell^{-} \bar{v}$ (with $\ell=e, \mu$ ) and, to a lesser extent $H \rightarrow \tau^{+} \tau^{-}$, come from the $g g$ fusion mechanism which is known to be affected by large theoretical uncertainties.
As a matter of fact, although the cross section $\sigma(g g \rightarrow$ $H$ ) is known up to next-to-next-to-leading order (NNLO) in perturbative QCD [24-31] (and at least at NLO for the electroweak interaction [32-37]), there is a significant residual scale dependence which points to the possibility that still higher order contributions beyond NNLO cannot be totally excluded. In addition, as the process is of $\mathcal{O}\left(\alpha_{s}^{2}\right)$ at $\mathrm{LO}$ and is initiated by gluons, there are sizable uncertainties due to the gluon parton distribution function (PDF) and the value of the coupling $\alpha_{s}$. In total, the combined theoretical uncertainty ${ }^{1}$ has been estimated to be of order $\Delta^{\text {th }} \sigma(g g \rightarrow H) \approx \pm 20 \%$ by the LHC Higgs cross section working group (LHCHWG) [22]. Hence, the theoretical uncertainty is already at the level of the accuracy of the measured cross section by the ATLAS and CMS collaborations, Eqs. (1)-(2).

The impact of the theoretical uncertainty can be viewed as follows [39]. The normalization cross section $\sigma^{\mathrm{SM}}$ adopted by the ATLAS and CMS collaborations, and which led to the results of Eqs. (1)-(2), is borrowed from the LHCHWG. For the dominant $g g \rightarrow H$ process, it is obtained by using the calculation performed at NNLO in QCD with a central choice for the renormalization and factorization scales $\mu_{F}=\mu_{R}=\mu_{0}=\frac{1}{2} M_{H}$ (which is approximately the same as what is obtained using the resummed cross section at next-to-next-to-leading-logarithm with a central scale $\mu_{0}=M_{H}$ ) and by adopting the MSTW2008 NNLO set of PDFs [40] to evaluate the gluon density, $\left.\sigma^{\mathrm{SM}} \simeq \sigma(g g \rightarrow H)\right|_{\mu_{0}=\frac{1}{2} M_{H}} ^{\mathrm{MSTW}}$. However, in principle, any NNLO PDF set and any scale choice in the conventional range $\frac{1}{2} \leq \mu / \mu_{0} \leq 2$ can be adopted to evaluate the cross

\footnotetext{
${ }^{1} \mathrm{~A}$ third source of theoretical uncertainties, the use of an effective field theory approach to calculate the radiative corrections beyond the NLO approximation, should in principle also be considered [23, 38] and would increase the total theoretical uncertainty up to $\Delta^{\text {th }} \sigma(g g \rightarrow$ $H) \approx \pm 25-30 \%$. In addition, large uncertainties arise when the $g g \rightarrow$ $H$ cross section is broken into jet categories as will be discussed later.
} 
section $\sigma(g g \rightarrow H)$, the difference compared to the reference value being accounted for by the theoretical uncertainty. For instance, if one uses the NNPDF PDF set [41] and adopts a scale choice $\mu_{0}=\frac{1}{4} M_{H}$, one would obtain a cross section that is $\approx 20 \%$ higher than the reference cross section

$\left.\sigma(g g \rightarrow H)\right|_{\mu_{0}=\frac{1}{4} M_{H}} ^{\mathrm{NNPDF}} \approx 1.21 \sigma^{\mathrm{SM}}$

In turn, if the cross section is evaluated with the ABM11 NNLO PDF set [42] with a scale choice $\mu_{0}=M_{H}$, one would have a cross section that is $\approx 15 \%$ lower,

$\left.\sigma(g g \rightarrow H)\right|_{\mu_{0}=M_{H}} ^{\mathrm{ABM}} \approx 0.85 \sigma^{\mathrm{SM}}$

Hence, if ATLAS had used the cross section value of Eq. (3) and CMS the one of Eq. (4) for the determination of the signal strength $\hat{\mu}$ of Eqs. (1)-(2), they both would have had a $2 \sigma$ discrepancy from the SM expectation. In particular, as was discussed in Ref. [39], the $\approx 2 \sigma$ excess observed by the ATLAS collaboration in the $H \rightarrow \gamma \gamma$ channel would turn into either a simple $1 \sigma$ or a tantalising $3 \sigma$ effect, depending on the chosen normalization.

It is therefore very important to eliminate this cross section normalization problem (or, in other words, the theoretical uncertainty) as it induces a bias that is already now, i.e. with the $\approx 10 \mathrm{fb}^{-1}$ data presently collected by ATLAS and CMS, at the level of the experimental accuracy of the cross section measurement. This uncertainty will be the principal limiting factor in the extraction of the Higgs couplings in a very near future.

A possibility which was considered to be very promising is the VBF production channel, $q q \rightarrow H q q$ with $H \rightarrow$ $W W, \gamma \gamma$ and $\tau \tau$, in which specific cuts reduce the various backgrounds significantly and allow a nice extraction of the Higgs signal [43-47]. Indeed, the cross section for the inclusive process has been shown to have a very small combined scale and PDF uncertainty, $\lesssim \pm 3 \%$ for $M_{H}=125 \mathrm{GeV}$ at $\sqrt{s}=7-8$ or $14 \mathrm{TeV}$ [22]. However, it turns out that at least at $\sqrt{s}=7-8 \mathrm{TeV}$, the process is contaminated by a significant fraction of gluon fusion events, $g g \rightarrow H+j j$, of the order of $30 \%$ [48-52], even after the specific cuts that select the VBF configuration are applied. The $g g \rightarrow H+j j$ channel is affected by much larger uncertainties than the inclusive $g g \rightarrow H$ process, up to $50 \%$ when one adds the scale and the PDF uncertainties, as well as by additional uncertainties from the jet veto when $\sigma(g g \rightarrow H)$ is broken into jet cross sections [53-56]. This makes the total uncertainty in the $H+j j$ final sample that includes the VBF part as large as in the inclusive $g g \rightarrow H$ case.

To remove the theoretical (and other) uncertainties, we suggest in this paper to simply consider ratios of production cross sections times decay branching fractions. Similar ratios have been proposed in the past, in particular by
D. Zeppenfeld and collaborators [57-60], at a time when the $g g \rightarrow H \rightarrow Z Z, W W$ channels were not expected to be viable for Higgs masses below $130 \mathrm{GeV}$ and the focus was on the vector boson fusion processes which appeared to be more promising [43-47]. Here, we first extend on these previous analyses by including all channels that are expected to be observable at the LHC, in particular those in which the $g g \rightarrow H$ cross section is broken into jet categories and the modes $g g \rightarrow H \rightarrow \tau^{+} \tau^{-}$and $q \bar{q} \rightarrow H V$ with boosted $H \rightarrow b \bar{b}$, which have not been considered in Refs. [57-60]. A generalized formalism for decay ratios that are free of theory uncertainties, denoted by $D_{X X}$, and also cross section ratios $C_{X X}$ which are less powerful as they still involve these uncertainties, is introduced. For an illustration of how the proposed ratios could be used in practice, we perform a rough analysis of the present ATLAS and CMS data and conclude that there is, presently, no significant deviation of these ratios from the SM expectation.

\section{The decay ratios $D_{X X}$}

To define ratios of Higgs production cross sections, one needs first to chose a reference channel for the Higgs decays. The most obvious ones are the modes $H \rightarrow V V^{*}$ with $V=Z, W$, which lead to the clean $H \rightarrow Z Z^{*} \rightarrow 4 \ell^{ \pm}$or $H \rightarrow W W^{*} \rightarrow \ell \ell v v$ final states. One then defines the decay ratio, which we denote by $D_{X X}$, for a given search channel $H \rightarrow X X$,

$$
\begin{aligned}
D_{X X} & =\frac{\sigma(g g \rightarrow H \rightarrow X X)}{\sigma(g g \rightarrow H \rightarrow V V)} \\
& =\frac{\sigma(g g \rightarrow H) \times \mathrm{BR}(H \rightarrow X X)}{\sigma(g g \rightarrow H) \times \mathrm{BR}(H \rightarrow V V)} \\
& =\frac{\Gamma(H \rightarrow X X)}{\Gamma(H \rightarrow V V)}
\end{aligned}
$$

in which the cross section $\sigma(g g \rightarrow H)$ and hence, its significant theory uncertainty, cancels out, ${ }^{2}$ leaving only the ratio of decay branching fractions and hence of partial decay widths. In fact, even the total Higgs decay width, which includes the contributions of channels such as $H \rightarrow g g$ and $H \rightarrow c \bar{c}$ that cannot be accessed at the LHC, as well as possible invisible Higgs decays in scenarios beyond the SM, do not appear in the decay ratios $D_{X X}$.

In addition, two other important sources of experimental uncertainties also cancel out: some common experimental systematical uncertainties such as, for instance, the one due to the luminosity measurement which is presently at

\footnotetext{
${ }^{2}$ When considering the same production process, a shift in the normalization should be noticed in the correlation between two different decay channels; we thank Dieter Zeppenfeld for a discussion on this point.
} 
Table 1 The reduced decay ratios $d_{X X}$ for the various final states $H \rightarrow X X$ observable at the LHC depending on the channel used as normalization, $H \rightarrow Z Z, W W$ or $V V=Z Z+W W$. These numbers are for $M_{H}=125 \mathrm{GeV}$ and are obtained using the program HDECAY [63] for the Higgs branching ratios when the SM inputs recommended by the LHCHWG [22] are used

\begin{tabular}{llllclll}
\hline Normalization & $d_{W W}$ & $d_{Z Z}$ & $d_{\tau \tau}$ & $d_{b b}$ & $d_{\gamma \gamma}$ & $d_{\mu \mu}$ & $d_{\gamma Z}$ \\
\hline$H \rightarrow Z Z$ & 8.14 & 1 & 2.39 & 21.9 & $8.64 \times 10^{-2}$ & $8.33 \times 10^{-3}$ & $5.72 \times 10^{-2}$ \\
$H \rightarrow W W$ & 1 & 0.123 & 0.294 & 2.68 & $1.06 \times 10^{-2}$ & $1.02 \times 10^{-3}$ & $0.72 \times 10^{-2}$ \\
$H \rightarrow V V$ & 0.89 & 0.11 & 0.261 & 2.39 & $0.94 \times 10^{-2}$ & $0.91 \times 10^{-3}$ & $0.64 \times 10^{-2}$ \\
\hline
\end{tabular}

the level of a few percent, and the uncertainties in the Higgs branching ratios which are of the order of 3-5\% for $\operatorname{BR}(H \rightarrow b \bar{b}, W W, Z Z, \gamma \gamma, \tau \tau)$. The latter uncertainties are mainly due to the $H \rightarrow b \bar{b}$ partial decay width which is affected by the errors on the input values of the bottom quark mass and the coupling $\alpha_{s}$ and which then migrate to the decays branching fractions through the total Higgs decay width that is controlled by the $b \bar{b}$ mode [23, 61]. In the decay ratios of Eq. (5), these uncertainties disappear when one considers final states like $H \rightarrow W W, Z Z, \gamma \gamma, \tau \tau$, where, in contrast to Higgs decays into quark and gluon pairs, only small electroweak effects are involved and no significant uncertainty in the partial widths occurs. ${ }^{3}$

Doing so, one would have the following theoretically "clean" observables to consider:

$$
\begin{aligned}
D_{Z Z} & =\frac{\sigma(g g \rightarrow H \rightarrow Z Z)}{\sigma(g g \rightarrow H \rightarrow V V)}=\frac{\Gamma(H \rightarrow Z Z)}{\Gamma(H \rightarrow V V)} \\
& =d_{Z Z} \frac{c_{Z}^{2}}{c_{V}^{2}} \\
D_{W W} & =\frac{\sigma(g g \rightarrow H \rightarrow W W)}{\sigma(g g \rightarrow H \rightarrow V V)}=\frac{\Gamma(H \rightarrow W W)}{\Gamma(H \rightarrow V V)} \\
& =d_{W W} \frac{c_{W}^{2}}{c_{V}^{2}} \\
D_{\tau \tau} & =\frac{\sigma(g g \rightarrow H \rightarrow \tau \tau)}{\sigma(g g \rightarrow H \rightarrow V V)}=\frac{\Gamma(H \rightarrow \tau \tau)}{\Gamma(H \rightarrow V V)} \\
= & d_{\tau \tau} \frac{c_{\tau}^{2}}{c_{V}^{2}} \\
D_{\gamma \gamma}= & \frac{\sigma(g g \rightarrow H \rightarrow \gamma \gamma)}{\sigma(g g \rightarrow H \rightarrow V V)}=\frac{\Gamma(H \rightarrow \gamma \gamma)}{\Gamma(H \rightarrow V V)} \\
= & d_{\gamma \gamma} \frac{c_{\gamma}^{2}}{c_{V}^{2}}
\end{aligned}
$$

\footnotetext{
${ }^{3}$ Note that in the case of the three-body $H \rightarrow W W^{*}$ and $H \rightarrow Z Z^{*}$ decay channels, the virtuality of the off-shell gauge bosons is important and the Higgs mass $M_{H}$, on which the partial decay widths depend crucially as can be seen from Fig. 1, has to be very precisely known.
}

where $c_{X}$ are the Higgs couplings to the $X$ states normalized to their SM values, ${ }^{4} c_{X} \equiv g_{H X X} / g_{H X X}^{\mathrm{SM}}$, and the reduced decay width ratios $d_{X X}$, which involve only gauge couplings and kinematical factors, are displayed in Table 1 for the three possible normalizations.

At this stage, a few important remarks are in order.

(i) To the observables of Eqs. (6)-(9), one could add $D_{\mu \mu}$ and $D_{Z \gamma}$ for the channels $H \rightarrow \mu^{+} \mu^{-}$and $H \rightarrow Z \gamma$ which could be measured with some accuracy at the upgrade of the LHC when $\sqrt{s} \approx 14 \mathrm{TeV}$ is reached and a large luminosity, $\gtrsim 100 \mathrm{fb}^{-1}$, is collected. The corresponding $d_{\mu \mu}$ and $d_{\gamma Z}$ decay factors are also given in Table 1 for completeness.

(ii) For the loop induced $H \rightarrow \gamma \gamma$ channel (the situation is similar in the case of $H \rightarrow Z \gamma$ ), we have defined the reduced coupling $c_{\gamma}$. In the SM, this decay is mediated by loops involving mainly the $W$ boson and the heavy top quark, with subleading contributions from the $b, c$ quarks and the $\tau$-lepton. In beyond the SM scenarios, not only the reduced couplings $c_{W}$ and $c_{f}$ are altered, but also new particles could contribute to the loops. For $M_{H} \approx 125 \mathrm{GeV}$ and retaining only the dominant $W$ and $t$ contributions, the $c_{\gamma}$ coupling where $\hat{c}_{\gamma}$ represents the possible contribution of new physics, can be written as

$c_{\gamma} \approx 1.26 \times\left|c_{W}-0.21 c_{t}+\hat{c}_{\gamma}\right|$

(iii) We do not include the branching fractions for the $Z \rightarrow$ $\ell^{-} \ell^{+}$and $W \rightarrow \ell v$ decays which are precisely known [64]. In fact, in the $H \rightarrow Z Z$ and $H \rightarrow W W$ decays, one could also include other channels such as $H \rightarrow Z Z \rightarrow \ell \ell v \bar{v}$ and $H \rightarrow W W \rightarrow \ell v j j$ if, in the future, they turn out to be useful for a $\approx 125 \mathrm{GeV}$ Higgs boson.

(iv) Finally, the decay ratios $D_{W W}$ or $D_{Z Z}$, depending on the chosen normalization, are proportional to the ratio of

\footnotetext{
${ }^{4}$ We will assume $c_{X}$ to be simply (real) constants and do not consider anomalous vertices with, for instance, derivative Higgs couplings to fermions or gauge bosons. The kinematics of the processes and the selection efficiencies are thus the same as in the SM. Derivative (momentum-dependent) Higgs couplings can be checked, for instance, by evaluating the production cross sections at different c.m. energies [62].
} 
squared couplings $c_{W}^{2} / c_{Z}^{2}$ and test custodial symmetry. They are thus related to the Veltman $\rho$ parameter [65] or, equivalently, to the Peskin-Takeuchi $T$ [66] or Altarelli-Barbieri $\epsilon_{1}$ [67] parameters,

$c_{W}^{2} / c_{Z}^{2} \approx \rho \approx M_{W}^{2} /\left(\cos ^{2} \theta_{W} M_{Z}^{2}\right) \approx 1+\alpha T \approx 1+\epsilon_{1}$

which from the high precision electroweak data has been shown to be very close to unity ${ }^{5}$ [64]. Assuming custodial symmetry, one could then assume $c_{W}=c_{Z}=c_{V}$ and use the combined $H \rightarrow W W$ and $H \rightarrow Z Z$ channels,

$$
\Gamma(H \rightarrow V V)=\Gamma(H \rightarrow W W)+\Gamma(H \rightarrow Z Z)
$$

as a reference channel in Eqs. (6)-(9), to increase the statistical accuracy of the normalization factor. The reduced decay values $d_{X X}$ in this case are also given in Table 1.

The previous discussion assumes that one can consider only the dominant $g g \rightarrow H$ production channel. Nevertheless, in practice, the other processes, in particular the vector boson fusion and the associated HV channels, contribute also to the total Higgs cross section and, more importantly, these channels lead to specific topologies that greatly facilitates the Higgs search in some cases. One should thus use a more accurate expression compared to Eq. (5) to define the decay ratios $D_{X X}$. Ignoring the $t \bar{t} H$ production channel for the moment (but its small contribution can be readily included), one would then have

$$
\begin{aligned}
D_{X X} & =\frac{\epsilon_{g g}^{X} \sigma(g g \rightarrow H \rightarrow X X)+\epsilon_{V B F}^{X} \sigma(q q \rightarrow H q q \rightarrow q q X X)+\epsilon_{H V}^{X} \sigma(q \bar{q} \rightarrow V H \rightarrow V X X)}{\epsilon_{g g}^{V} \sigma(g g \rightarrow H \rightarrow V V)+\epsilon_{V B F}^{V} \sigma(q q \rightarrow H q q \rightarrow q q V V)+\epsilon_{H V}^{V} \sigma(q \bar{q} \rightarrow V H \rightarrow V V V)} \\
& =\frac{\epsilon_{g g}^{X} \sigma(g g \rightarrow H)+\epsilon_{V B F}^{X} \sigma(q q \rightarrow H q q)+\epsilon_{H V}^{X} \sigma(q \bar{q} \rightarrow V H)}{\epsilon_{g g}^{V} \sigma(g g \rightarrow H)+\epsilon_{V B F}^{V} \sigma(q q \rightarrow H q q)+\epsilon_{H V}^{V} \sigma(q \bar{q} \rightarrow V H)} \times \frac{\Gamma(H \rightarrow X X)}{\Gamma(H \rightarrow V V)}
\end{aligned}
$$

where $\epsilon^{X}$ stands for the experimental efficiency to select the Higgs events in the $g g$, VBF, and HV channels. Note that we left the normalization channel $H \rightarrow V V$ unspecified in such a way that any of the $Z Z, W W$ or $V V=W W+Z Z$ possibilities can be chosen. The second line of Eq. (13) shows that still, some common systematical uncertainties and the uncertainties in the Higgs branching ratio cancel out. In addition, if the efficiencies $\epsilon^{X}$ and $\epsilon^{V}$ are comparable or one production channel is dominant, a large part of the cross section uncertainties also cancel out. There is thus still a clear advantage in using these ratios.

In fact, since the VBF and the HV channels involve two additional jets (or leptons in the case of HV) in the final state, the $g g$ fusion mechanism can be singled out by considering the Higgs +0 jet cross sections, i.e. by requiring that no hard jet (with $p_{T}^{\text {jet }}$ larger than say $30-40 \mathrm{GeV}$ ) is produced along with the Higgs particle. One can have then almost pure $g g$ fusion events and construct the ratio,

\footnotetext{
${ }^{5}$ In addition, only very few new physics models (e.g. models with Higgs triplets and some composite models) allow for deviation of this ratio for unity at tree-level; for a recent discussion, see Ref. [68]. We also note that if custodial symmetry is violated, the parameters $\rho$ etc. of Eq. (11) become sensitive to the ultraviolet cut-off and the equation becomes questionable.
}

$D_{X X}^{(0 j)}=\frac{\sigma(g g \rightarrow H+0 j \rightarrow X X)}{\sigma(g g \rightarrow H+0 j \rightarrow V V)}=\frac{\Gamma(H \rightarrow X X)}{\Gamma(H \rightarrow V V)}$

As the additional jets are produced at higher orders in QCD, NLO or NNLO, the Higgs +0 jet cross sections represent a large fraction of the $g g \rightarrow H$ inclusive rate: for $p_{T}^{\text {jet }} \gtrsim$ $30 \mathrm{GeV}$, one has very crudely $60 \%, 30 \%$ and $10 \%$ for, respectively, the 0,1 , and 2 jet cross sections. However, as was recently realized, the breaking of the Higgs cross sections into jet categories introduces significant uncertainties [5356]. These additional uncertainties, if one adopts the same criteria (same jet veto etc.) for selecting the $X X$ and the $V V$ events in the numerator and denominator of Eq. (14), will also cancel out in the ratio.

Another remark is that one can also include to the $\mathrm{H}+$ $1 j$ contributions in Eq. (14), and thus consider the ratios $D_{X X}^{(0+1 j)}$. This would increase the number of signal events without having too much contamination from the VBF and $\mathrm{VH}$ processes. However, in the channel $H \rightarrow W W \rightarrow$ $\ell \ell v v+1 j$ for instance, one would have to deal with the large $t \bar{t} \rightarrow b \bar{b} W W$ background in which one of the (untagged) $b-$ jets is soft and escapes detection.

In turn, if one focuses on the VBF events which have a special topology and, in most cases, have a more favorable signal to background ratio [43-47], one would consider the ratio 


$$
\begin{aligned}
D_{X X}^{(2 j)} & =\frac{\epsilon_{g g}^{X} \sigma(g g \rightarrow H j j \rightarrow X X j j)+\epsilon_{V B F}^{X} \sigma(q q \rightarrow H q q \rightarrow q q X X)+\epsilon_{H V}^{X} \sigma(q \bar{q} \rightarrow V H \rightarrow q \bar{q} X X)}{\epsilon_{g g}^{V} \sigma(g g \rightarrow H j j \rightarrow V V j j)+\epsilon_{V B F}^{V} \sigma(q q \rightarrow H q q \rightarrow q q V V)+\epsilon_{H V}^{V} \sigma(q \bar{q} \rightarrow V H \rightarrow q \bar{q} V V)} \\
& =\frac{\epsilon_{g g}^{X} \sigma(g g \rightarrow H j j)+\epsilon_{V B F}^{X} \sigma(q q \rightarrow H q q)+\epsilon_{H V}^{X} \sigma(q \bar{q} \rightarrow V H)}{\epsilon_{g g}^{V} \sigma(g g \rightarrow H j j)+\epsilon_{V B F}^{V} \sigma(q q \rightarrow H q q)+\epsilon_{H V}^{V} \sigma(q \bar{q} \rightarrow V H)} \times \frac{\Gamma(H \rightarrow X X)}{\Gamma(H \rightarrow V V)}
\end{aligned}
$$

While most of the events from the HV process can be removed by requiring that the jet-jet invariant mass does not coincide with $M_{W}$ or $M_{Z}$, a significant fraction of the $g g \rightarrow H+2 j$ events (of the order of $30 \%$ [48-52]) will remain even after the specific cuts that select the VBF configuration are applied. Hence, despite the very small scale and PDF uncertainty that affects the inclusive VBF Higgs cross section [22], the contamination by the $g g \rightarrow H+j j$ channel in which the combined scale + PDF uncertainty is at the level of $\approx 50 \%$, will make the total uncertainty in the $H+j j$ final sample very large. Again, by performing the ratio of Eq. (15), one could reduce, if not almost completely eliminate, the theoretical uncertainty in the extraction of the Higgs couplings from these processes.

One can also use the same procedure in the case of the Higgs +1 jet configuration which, for instance, can be appropriate in the $H \rightarrow \tau \tau$ search channel [69]. In fact, this configuration would be extremely useful in the case of invisible Higgs decays which can be searched for in monojet events in the process $g g \rightarrow H+1 j$ with $H \rightarrow$ invisible as has been recently discussed in Ref. [70] for instance. In this case, one could consider the ratio,

$$
\begin{aligned}
D_{\text {inv }}^{(1 j)} & =\frac{\sigma\left(g g \rightarrow H+1 j \rightarrow 1 j+\not \notin_{T}\right)}{\sigma(g g \rightarrow H+1 j \rightarrow 1 j+V V)} \\
& =\frac{\Gamma(H \rightarrow \text { inv })}{\Gamma(H \rightarrow V V)}
\end{aligned}
$$

in which, again, the theoretical uncertainties in the $g g \rightarrow$ $H$ cross section, which could mimic the additional invisible contribution to the total Higgs decay width, will cancel out.

Of course, ultimately, the ratios $D_{X X}$ for a given final state and from different jet configurations should be combined to reach a better statistical accuracy.

Let us now turn to the $H \rightarrow b \bar{b}$ final state which deserves a special treatment as it is observable mainly (if not exclusively) in the $q \bar{q} \rightarrow H V \rightarrow b \bar{b} V$ process using boosted jet techniques to isolate the $b \bar{b}$ events [71]. One can use the process as it is to measure $\mathrm{BR}(H \rightarrow b \bar{b})$ as the cross section $\sigma(q \bar{q} \rightarrow H V)$ is predicted with an accuracy of $\approx 5 \%$ [22] that will be much smaller than the experimental error. But one can also consider the ratio

$$
\begin{aligned}
D_{b b} & =\frac{\sigma(q \bar{q} \rightarrow H V \rightarrow b \bar{b} V)}{\sigma(q \bar{q} \rightarrow H V \rightarrow V V V)}=\frac{\Gamma(H \rightarrow b \bar{b})}{\Gamma(H \rightarrow V V)} \\
& =d_{b b} \frac{c_{b}^{2}}{c_{V}^{2}}
\end{aligned}
$$

However, as the cross sections times branching ratios for the clean $H \rightarrow Z Z \rightarrow 4 \ell$ or $H \rightarrow W W \rightarrow \ell \ell \nu v$ final states are very small, the normalization above might not be appropriate.

If the Higgs signal could be extracted in the $q \bar{q} \rightarrow$ $H V \rightarrow \tau^{+} \tau^{-} V$ channel (as, for instance, recently advocated in Ref. [75]), the situation would be rather straightforward as one could simply consider the ratio of $b \bar{b} V$ to $\tau^{+} \tau^{-} V$ production, $D_{b b / \tau}=\sigma(q \bar{q} \rightarrow H V \rightarrow b \bar{b} V) / \sigma(q \bar{q}$ $\rightarrow H V \rightarrow V \tau \tau)=\Gamma(H \rightarrow b \bar{b}) / \Gamma(H \rightarrow \tau \tau)$ which directly provides the important ratio $c_{b}^{2} / c_{\tau}^{2}$, which allows to test the hierarchy of the Higgs-fermion couplings and the important SM prediction, $c_{b}^{2} / c_{\tau}^{2} \approx 3 \bar{m}_{b}\left(M_{H}^{2}\right) / m_{\tau}^{2} \approx 10$.

\section{The cross section ratios $C_{X X}$}

So far, we have only considered a given production process with different decay channels and constructed decay ratios $D_{X X}$ in which the theoretical uncertainties in the cross sections as well as some model dependence due to the Higgs total decay width should cancel out. However, when doing so, some very important information that is contained in the cross section only has been removed. This was, for instance, the case of the Higgs to gluons coupling which generates the $g g \rightarrow H$ process. In this section, we briefly consider ratios of cross sections for different production processes but for a given Higgs decay channel in which this information is retained. In these cross section ratios, which we denote by $C_{X X}$, it is the branching fractions or the partial decay width (and hence the ambiguities in the total width) which will cancel out in addition to some systematical errors that are common to the two processes, leaving us only with the theoretical uncertainties due to the production cross sections. In the absence of cleaner ratios to probe the couplings involved in the cross sections in an almost model independent way, and in order not to lose the crucial information that they provide, this choice can be considered as a "lesser evil". 
We start by reconsidering the determination of the $\mathrm{Hbb}$ coupling from the HV process. Instead of performing the decay ratio of Eq. (17) in which the normalization might not be appropriate, one can take advantage of the fact that the HV and the VBF (inclusive) rates are affected by rather small theoretical uncertainties and consider the cross section ratio

$$
\begin{aligned}
C_{b b} & =\frac{\sigma(q \bar{q} \rightarrow H V \rightarrow b \bar{b} V)}{\sigma(q q \rightarrow H q q \rightarrow V V q q)} \propto \frac{\Gamma(H \rightarrow b \bar{b})}{\Gamma(H \rightarrow V V)} \\
& \propto \frac{c_{b}^{2}}{c_{V}^{2}}
\end{aligned}
$$

As the production cross sections in both processes are proportional to the square of the coupling $c_{V}$, it will cancel out in the ratio ${ }^{6}$ leaving only the dependence on the decay ratios (this is particularly the case if one assumes the custodial symmetry which enforces the relation $c_{W}=c_{Z}$ that simplifies the problem). Of course, different systematics will enter the two processes and they have to be taken care of. However, at least the uncertainties from the luminosity, the Higgs total width and eventually also part of the uncertainty due to the PDFs (as both processes are initiated by incoming quarks) will cancel out. The usual systematical experimental errors in the selection of the two channels as well as the very small scale uncertainty that affect the two processes remain though. This nice picture is, however, spoilt by the contamination of the VBF process by the $g g \rightarrow H j j$ contribution.

Another issue is the determination of the important Higgs coupling to top quarks. This coupling can be first determined indirectly from the $g g \rightarrow H$ cross section which, as discussed earlier, is dominantly generated by a top quark loop and hence is proportional to $g_{H t t}^{2}$. By normalizing to the VBF process, one would have the ratio

$$
C_{g g}=\frac{\sigma(g g \rightarrow H \rightarrow V V)}{\sigma(q q \rightarrow H q q \rightarrow V V q q)} \propto \frac{c_{g}^{2}}{c_{V}^{2}}
$$

which nevertheless includes the large theoretical uncertainty that affects the $g g \rightarrow H$ rate and, eventually, the comparable one of VBF if the contamination by $g g \rightarrow H j j$ events remains large. One can chose a decay normalization with $V=W, Z$ or $W+Z$ but one could also add the case $V=\gamma$ to increase the statistics; the $H \gamma \gamma$ coupling, which is also very sensitive to new physics, will anyway drop in the ratio. The coupling $c_{g}$ receives a dominant contribution from the top quark, but also a smaller one from the bottom quark; contributions from new strongly interacting particles are also possible (see also Ref. [9]):

$c_{g} \approx 1.075 \times\left|c_{t}-(0.066+0.093 i) c_{b}+\hat{c}_{g}\right|$

\footnotetext{
${ }^{6}$ Note that in $\sigma(p p \rightarrow H Z)$, there is a contribution from the $g g \rightarrow H Z$ box diagram which is not proportional to $c_{V}^{2}$ [72-74] and which is about $5-10 \%$ depending on the considered c.m. energy.
}

Nevertheless, one should consider the previous ratio as a measurement of the Higgs to gluons coupling, rather than the $H t \bar{t}$ coupling, as there is the possibility of loop contributions from new strongly interacting particles that couple to the Higgs boson.

A more direct measurement of the $H t \bar{t}$ coupling can be performed in the $p p \rightarrow t \bar{t} H$ process, once enough data is collected. In this case, one could consider the ratio ${ }^{7} C_{t t}=$ $\sigma(p p \rightarrow H t \bar{t} \rightarrow t \bar{t} V V) / \sigma(q q \rightarrow H q q \rightarrow V V q q)$ which is proportional to $c_{t}^{2} / c_{V}^{2}$. However, as the most interesting process in this context (if it is made viable experimentally) is $p p \rightarrow H t \bar{t}$ with $H \rightarrow b \bar{b}$, the proper normalization to use should be the $q \bar{q} \rightarrow V H \rightarrow V b \bar{b}$ channel

$C_{t t}=\frac{\sigma(p p \rightarrow H t \bar{t} \rightarrow t \bar{t} b \bar{b})}{\sigma(q \bar{q} \rightarrow V H \rightarrow V b \bar{b})} \propto \frac{c_{t}^{2}}{c_{V}^{2}}$

In both cases Eqs. (19) and (21), the branching fractions and some common systematical errors have canceled out, leaving us only with the theoretical uncertainties due to the $g g \rightarrow H$ and $q \bar{q} / g g \rightarrow t \bar{t} H$ production cross sections, as the one affecting at least the (inclusive) HV channel is particularly small.

A final word is due to the Higgs self-coupling which, at the LHC, can be only determined from double Higgs production in the $g g \rightarrow H H$ process once a very high luminosity is collected [76]. As the process is initiated by gluons similarly to the $g g \rightarrow H$ case, and the NLO QCD corrections in both processes are very similar [77], a large component of the QCD uncertainties should drop if one considers the ratio

$$
\begin{aligned}
C_{H H}= & \frac{\sigma(g g \rightarrow H H)}{\sigma(g g \rightarrow H)} \propto\left(a g_{H H H}+b g_{H t t}\right)^{2} \\
& \times \frac{\mathrm{BR}(H \rightarrow X X) \cdot \mathrm{BR}(H \rightarrow Y Y)}{\mathrm{BR}(H \rightarrow X X)}
\end{aligned}
$$

and one would be mostly left with the smaller branching fractions uncertainties.

\section{Application of the ratios to the LHC data}

It is clear that a truly reliable estimate of the experimental accuracies in the determination of the ratios of cross sections discussed previously can only come from the ATLAS and CMS collaborations as they have the full information on the systematical errors that affect their measurements and

\footnotetext{
${ }^{7}$ Note that in the $p p \rightarrow t \bar{t} H$ process, the PDF uncertainty is the largest source of error and is about $\pm 10 \%$ for $M_{H}=125 \mathrm{GeV}$ at $\sqrt{s}=14 \mathrm{TeV}$ [22]. One could reduce this uncertainty by normalizing the cross section to the $p p \rightarrow t \bar{t}$ rate, eventually at high enough invariant $t \bar{t}$ mass to be in the same kinematical regime as in the $p p \rightarrow t \bar{t} H$ process. We thank R. Godbole for a discussion on this point.
} 
Table 2 The ATLAS and CMS signal strength modifiers $\hat{\mu}$ in the various search channels that are used for our illustration; they are obtained from Refs. $[6,7,78]$

\begin{tabular}{lllll}
\hline & $\hat{\mu}_{W W}$ & $\hat{\mu}_{Z Z}$ & $\hat{\mu}_{\gamma \gamma}$ & $\hat{\mu}_{\gamma \gamma j j}$ \\
\hline ATLAS & $1.3 \pm 0.5$ & $1.4 \pm 0.6$ & $1.8 \pm 0.5$ & $2.7 \pm 1.3$ \\
CMS & $0.67 \pm 0.4$ & $0.72 \pm 0.5$ & $1.6 \pm 0.5$ & - \\
\hline
\end{tabular}

the experimental efficiencies to select the various observed channels. Nevertheless, in order to illustrate the usefulness of the ratios that we have introduced in this paper, we will attempt in this section to provide a rough estimate of the accuracies that can be obtained on some of these ratios, using the partial information that was provided in the combined ATLAS [6] and CMS [7] analyses.

The ATLAS collaboration has given the best fit values and the corresponding uncertainties of the signal strength $\hat{\mu}$ in the inclusive search channels $H \rightarrow W W^{*} \rightarrow \ell \ell \nu v, Z Z^{*}$ $\rightarrow 4 \ell$ and $\gamma \gamma$ when the values at $\sqrt{s}=7$ and $8 \mathrm{TeV}$ are combined for $M_{H}=126 \mathrm{GeV}$ (Table 7 of Ref. [6]); these $\hat{\mu}$ values are listed in Table 2. Instead, the CMS collaboration did not provide the exact $\hat{\mu}$ values in these channels but reported them in a figure for $M_{H}=125.5 \mathrm{GeV}$ (Fig. 19 of Ref. [7]); the numbers listed in Table 2 are thus approximate. The exact values of $\hat{\mu}_{\gamma \gamma j j}$ for the $H \rightarrow \gamma \gamma$ channel in the VBF configuration have not be provided by both collaborations and the approximate one listed for ATLAS in Table 2 is taken from Fig. 14 of Ref. [78]. We do not consider the $H \rightarrow \tau \tau$ and $H \rightarrow b \bar{b}$ channels as, with the present data, the uncertainties are too still large.

We will first construct the cross section ratios in the inclusive search channels. For this purpose, we will make the following assumptions which seem to us rather reasonable: (i) the efficiencies for selecting the $H \rightarrow W W, Z Z, \gamma \gamma$ modes are approximately the same so that the cross section part in Eq. (13) drops out and we are left only with the ratios of partial decay widths; (ii) the uncertainties in the measurements are dominated by the statistical error as well as by systematical errors that are assumed to be uncorrelated in the different channels, can be treated as Gaussian (the theoretical and the common systematical errors from the luminosity and the Higgs branching ratios will drop in the ratios); (iii) the remaining uncertainties in the ATLAS and CMS results are assumed to be uncorrelated and the results of the two experiments can be averaged.

With these assumptions, we first construct the ratios $D_{Z Z}$ and $D_{W W}$, first for the individual experiments and, then, when the ATLAS and CMS results are averaged; to increase the statistics we use the normalization in which the channels $H \rightarrow Z Z$ and $H \rightarrow W W$ are combined. We obtain in this case, when averaging the ATLAS and CMS results (from now on and for simplicity, we will set the factors $d_{X X}$ given in Table 1 to unity)

$$
\begin{aligned}
& D_{W W} \equiv c_{W}^{2} / c_{V}^{2}=0.97 \pm 0.40 \\
& D_{Z Z} \equiv c_{Z}^{2} / c_{V}^{2}=1.04 \pm 0.46
\end{aligned}
$$

where the errors are only of experimental nature and mostly statistical. Remarkably, these values are already close to unity with the present data, showing that custodial symmetry approximately holds. ${ }^{8}$ At the end of this year, when $\approx 30 \mathrm{fb}^{-1}$ of data will hopefully be collected by both the ATLAS and the CMS experiments, custodial symmetry can be checked at the $\approx 25 \%$ level. Ultimately, if more than $300 \mathrm{fb}^{-1}$ of data is collected by the ATLAS and CMS collaborations at $\sqrt{s} \approx 14 \mathrm{TeV}$, these relations can be checked at the $\approx 5 \%$ level, with no limitation from theoretical uncertainties and hopefully also from systematical uncertainties if the two channels $H \rightarrow W W, Z Z$ are analyzed in the same way.

A second and extremely important ratio which can be already constructed from the ATLAS and CMS signal strength modifiers in the inclusive channels is $D_{\gamma \gamma}$. One obtains, again by combining the ATLAS and CMS results and using the combined $H \rightarrow V V=W W+Z Z$ channel as a normalization,

$D_{\gamma \gamma} \equiv c_{\gamma}^{2} / c_{V}^{2}=1.70 \pm 0.43$

which is in accord with the SM expectation at the $95 \%$ confidence level (CL). Again, this ratio should be free of theoretical and common systematical uncertainties and could be probed with an accuracy at the level of $25 \%$ at the end of this year and, ultimately, at the $5 \%$ level at the upgraded LHC. We expect this measurement to be the most important one to be performed in the Higgs sector at the LHC. It is crucial because first, it might involve contributions from new light charged particles that couple to the Higgs boson and second, because it measures the relative strength of the Higgs couplings to vector bosons and to the heavy top quark, Eq. (10), which is one of the most important checks of the Weinberg generalization [80] of the Englert-Brout-Higgs mechanism to fermions.

The left-hand panel of Fig. 2 shows the $68 \%$ CL contour in the $\left[c_{W}, c_{t}\right]$ plane that is allowed by the present data assuming custodial symmetry. Shown also are the contours that can be probed at $\sqrt{s}=8 \mathrm{TeV}$ with an integrated luminosity of $30 \mathrm{fb}^{-1}$ per experiment and at $\sqrt{s}=14 \mathrm{TeV}$ with $300 \mathrm{fb}^{-1}$ data, assuming again that the measurement will only be limited by statistics and that the central value stays

\footnotetext{
${ }^{8}$ Because we are combining the $H \rightarrow W W$ and $H \rightarrow Z Z$ channels for the normalization and the ATLAS and CMS $\hat{\mu}_{W W}$ and $\hat{\mu}_{Z Z}$ values, our result is more accurate than the one given by the CMS collaboration, $c_{W}^{2} / c_{Z}^{2} \equiv R_{W W / Z Z}=0.9_{-0.6}^{+1.1}[5]$.
} 
Fig. 2 The $68 \%$ CL contours for the couplings $c_{W}, c_{t}$ as presently allowed from the measurement of the ratio $D_{\gamma \gamma}$ at the LHC (left) and the $68 \% \mathrm{CL}$ allowed range in the plane $\left[C_{g g}^{-1}, D_{\gamma \gamma}\right]$ with and without the theoretical uncertainty $($ right). The prospects for $\sqrt{s}=8 \mathrm{TeV}$ and $30 \mathrm{fb}^{-1}$ data and $\sqrt{s}=14 \mathrm{TeV}$ and $300 \mathrm{fb}^{-1}$ data are also displayed in both cases
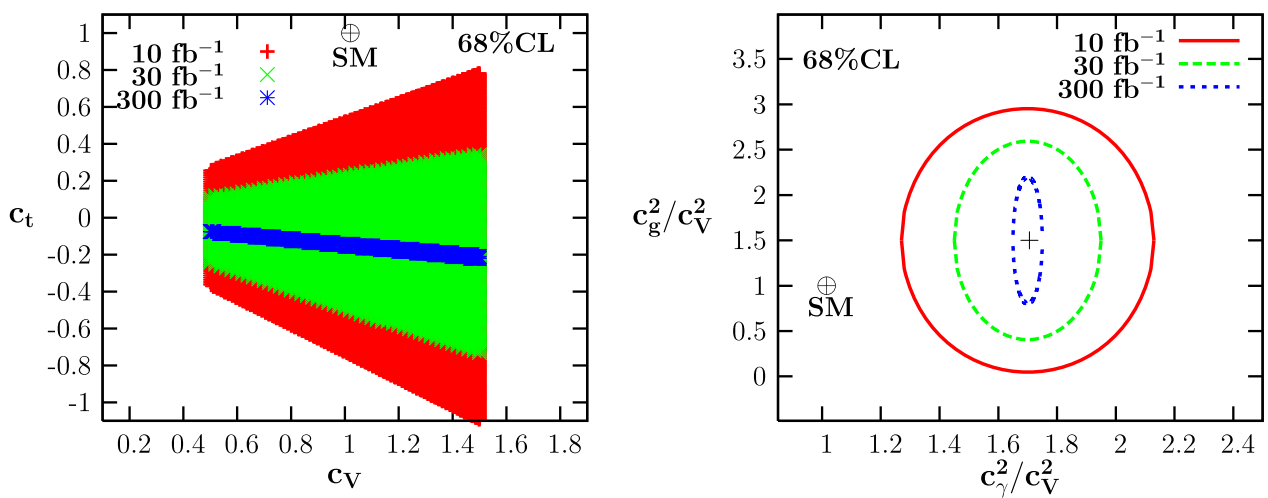

the same as presently. The few percent accuracy on the relative Higgs couplings $c_{W}$ and $c_{t}$ that can be achieved at the end of the planed LHC run is not only due to the increase of the luminosity to $300 \mathrm{fb}^{-1}$ but, also, to the increase of the $g g \rightarrow H$ inclusive cross section by a factor $\approx 2.5$ when increasing the energy from $8 \mathrm{TeV}$ to $14 \mathrm{TeV}$. Note that in the absence of new physics contributions $\hat{c}_{\gamma}$ to the $H \gamma \gamma$ coupling as is assumed in Fig. 2, the $c_{t}$ values which best fit the data are negative to accommodate the presently significant deviation of $D_{\gamma \gamma}$ from unity.

The other measurement which could be already exploited is the cross section for the VBF-like associated production of $\gamma \gamma$ events with dijets. In this case, the exact values of the strength modifier $\hat{\mu}_{\gamma \gamma j j}$ have not been given by both collaborations $^{9}$ and the approximate one that we quote for ATLAS in Table 2 is obtained from Fig. 14 of Ref. [78] with $M_{H}=126.5 \mathrm{GeV}$. Besides the experimental uncertainty of $50 \%$, we will assume an additional theoretical uncertainty of $\approx 20 \%$ which is mainly due to the contamination of the VBF channel by the $\approx 30 \% g g \rightarrow H+j j$ contribution which is affected by a $\approx 50 \%$ uncertainty as was discussed previously. This gives a value $\hat{\mu}_{\gamma \gamma j j}=2.7 \pm 1.3 \pm 0.5$ in which the two uncertainties are to be added linearly since the theoretical uncertainty has no statistical ground and should be considered as a bias rather than a mere nuisance.

Nevertheless, one cannot use the full potential of this measurement for the time being as there is no precise result for a corresponding measurement of the VBF $H \rightarrow$ $W W, Z Z$ or $\tau \tau$ channels to perform the ratios of Eq. (15) in which the large theoretical uncertainties cancel out and which, for instance, could be combined with $D_{\gamma \gamma}$ in Eq. (24)

\footnotetext{
${ }^{9}$ In Ref. [79], the CMS collaboration provided some numbers (again in a plot) for two configurations of the $\gamma \gamma$-dijet sample, with loose and tight cuts on the dijets, and separately for $\sqrt{s}=7$ and $8 \mathrm{TeV}$. Since we do not have all the information, we refrain from combining the numbers to obtain a global value for the strength modifier in this case. We emphasize again the fact that these numbers are very important (in contrast to the ones for the boosted decision trees which are irrelevant for our purpose) and should be part of the public information that is provided by the ATLAS and CMS experiments.
}

to reach a better level of accuracy. Hence, one can use $\hat{\mu}_{\gamma \gamma j j}$ as it is, but we prefer again to construct a ratio, the one of Eq. (19), to eliminate possible ambiguities from the Higgs branching fractions or the total Higgs decay width. The price to pay is the introduction of the large theoretical uncertainty that affects the inclusive $g g \rightarrow H$ cross section, which we take to be $\pm 20 \%$, and which need to be added to the one affecting the VBF-like cross section. This will allow to determine in a non-ambiguous way the ratio of couplings $c_{g}^{2} / c_{V}^{2}$, which provides additional interesting information. One obtains using the ATLAS result only

$C_{g g}^{-1}=\frac{\hat{\mu}_{\gamma \gamma j j}}{\hat{\mu}_{\gamma \gamma}}=c_{V}^{2} / c_{g}^{2}=1.5 \times[1 \pm 0.57 \pm 0.4]$

where the first uncertainty is experimental and the second one theoretical. This ratio is confronted to $D_{\gamma \gamma}$ in the righthand side of Fig. 2 where the $68 \%$ CL contour $\left[C_{g g}^{-1}, D_{\gamma \gamma}\right]$ is displayed for the present data, as well as for the projections for the end of this year and the end of the LHC run. One can see that while the error on $D_{\gamma \gamma}$ shrinks considerably when more data is collected, as it is mainly due to statistics, the total error on $C_{g g}^{-1}$ will be limited to the $40 \%$ theoretical uncertainty which can be reduced only with a more refined calculation of the $g g \rightarrow H$ cross section in the various jet categories.

Instead, when more data will be collected by ATLAS and CMS, other Higgs decay channels could be probed in the VBF configuration and ratios such as $D_{\gamma \gamma}^{(j j)}$ and $D_{\tau \tau}^{(j j)}$ could be then determined and would allow a very precise measurement of ratios of Higgs couplings in a way that is complementary to what is obtained in the inclusive $g g \rightarrow H$ mode. The power of the VBF mechanism could then be fully exploited.

\section{Conclusion}

We suggest to use ratios of Higgs production cross sections at the LHC for different Higgs decay channels such as $H \rightarrow$ 
$W W, Z Z, \tau \tau, b \bar{b}, \gamma \gamma$ and eventually $H \rightarrow \mu^{+} \mu^{-}, Z \gamma$, to determine the Higgs couplings to fermion and gauge bosons in a way that is not limited by theoretical uncertainties. These uncertainties, which are large being of order $\approx 20 \%$, affect not only the main production channel, $g g \rightarrow H$, but also the vector boson fusion channel $q q \rightarrow H q q$ as it is significantly contaminated by the $g g \rightarrow H j j$ contribution. The observables $D_{X X}$ that we propose involve ratios of Higgs partial decay widths and are hence also free from some systematical errors, such as the one from the luminosity measurement, and from other theoretical ambiguities such as those involved in the Higgs branching ratios or total decay width. In this respect, there is less model dependence in these ratios when beyond the SM scenarios are considered since they do not involve the Higgs total width.

One can also construct ratios of cross sections for different production processes with a given Higgs decay, $C_{X X}$, in which some ambiguities drop out, but these are less powerful than the ratios $D_{X X}$ as the theoretical uncertainties that affect the cross sections remain.

A rough analysis with the $\approx 10 \mathrm{fb}^{-1}$ data collected by ATLAS and CMS shows that some of these ratios are compatible with the SM expectation. At the end of the LHC run with $\sqrt{s}=14 \mathrm{TeV}$ and $\approx 300 \mathrm{fb}^{-1}$ data per experiment, some ratios can be determined with a very high accuracy, at the $5 \%$ level, without any limitation from theoretical uncertainties.

Hence, the LHC could become a precision machine for Higgs physics provided that ratios of cross sections times branching fractions for the same production channel, with eventually the same selection cuts for the different final state topologies, are considered.

Acknowledgements I thank the CERN Theory Unit for hospitality and J. Baglio, A. Falkowski, R. Godbole, C. Grojean, B. Mellado, and D. Zeppenfeld for discussions.

Open Access This article is distributed under the terms of the Creative Commons Attribution License which permits any use, distribution, and reproduction in any medium, provided the original author(s) and the source are credited.

\section{References}

1. P. Higgs, Phys. Lett. 12, 132 (1964)

2. P. Higgs, Phys. Rev. Lett. 13, 506 (1964)

3. F. Englert, R. Brout, Phys. Rev. Lett. 13, 321 (1964)

4. G. Guralnik, C. Hagen, T. Kibble, Phys. Rev. Lett. 13, 585 (1964)

5. F. Gianotti, J. Incandela for the ATLAS and CMS collaborations at the CERN meeting. Latest update in the search for the Higgs boson, 04/07/2012

6. ATLAS Collaboration, arXiv:1207.7214 [hep-ex]

7. CMS Collaboration, arXiv:1207.7235 [hep-ex]

8. D. Carmi, A. Falkowski, E. Kuflik, T. Volansky, J. Zupan, arXiv: 1207.1718 [hep-ph]

9. J. Espinosa, C. Grojean, M. Muhlleitner, M. Trott, arXiv: 1207.1717 [hep-ph]
10. P. Giardino, K. Kannike, M. Raidal, A. Strumia, arXiv:1207.1347 [hep-ph]

11. J. Ellis, T. You, arXiv:1207.1693 [hep-ph]

12. T. Corbett et al., arXiv:1207.1344 [hep-ph]

13. F. Bonnet, T. Ota, M. Rauch, W. Winter, arXiv:1207.4599 [hep$\mathrm{ph}]$

14. A. Alves et al., arXiv: 1207.3699 [hep-ph]

15. S. Banerjee, S. Mukhopadhyay, B. Mukhopadhyaya, arXiv:1207. 3588 [hep-ph]

16. A. Arbey et al., arXiv:1207.1348 [hep-ph]

17. I. Low, J. Lykken, G. Shaughnessy, arXiv:1207.1093 [hep-ph]

18. M. Klute et al., arXiv:1205.2699 [hep-ph]

19. M. Peskin, arXiv:1207.2516v1 [hep-ph]

20. T. Plehn, M. Rauch, arXiv:1207.6108 [hep-ph]

21. A. Djouadi, Phys. Rep. 457, 1 (2008)

22. S. Dittmaier et al., Handbook of LHC Higgs cross sections. arXiv:1101.0593 [hep-ph]

23. J. Baglio, A. Djouadi, J. High Energy Phys. 1103, 055 (2011). arXiv:1012.0530 [hep-ph]

24. H. Georgi, S.L. Glashow, M. Machacek, D.V. Nanopoulos, Phys. Rev. Lett. 40, 692 (1978)

25. A. Djouadi, M. Spira, P. Zerwas, Phys. Lett. B 264, 440 (1991)

26. S. Dawson, Nucl. Phys. B 359, 283 (1991)

27. M. Spira, A. Djouadi, D. Graudenz, P.M. Zerwas, Nucl. Phys. B 453, 17 (1995)

28. R.V. Harlander, W. Kilgore, Phys. Rev. Lett. 88, 201801 (2002)

29. C. Anastasiou, K. Melnikov, Nucl. Phys. B 646, 220 (2002)

30. V. Ravindran, J. Smith, W.L. Van Neerven, Nucl. Phys. B 665, 325 (2003)

31. S. Catani, D. de Florian, M. Grazzini, P. Nason, J. High Energy Phys. 0307, 028 (2003)

32. A. Djouadi, P. Gambino, Phys. Rev. Lett. 73, 2528 (1994)

33. A. Djouadi, P. Gambino, B.A. Kniehl, Nucl. Phys. B 523, 17 (1998)

34. U. Aglietti et al., Phys. Lett. B 595, 432 (2004)

35. G. Degrassi, F. Maltoni, Phys. Lett. B 600, 255 (2004)

36. S. Actis et al., Phys. Lett. B 670, 12 (2008)

37. C. Anastasiou, R. Boughezal, F. Pietriello, J. High Energy Phys. 0904, 003 (2009)

38. J. Baglio, A. Djouadi, J. High Energy Phys. 1010, 064 (2010)

39. J. Baglio, A. Djouadi, R. Godbole, arXiv:1207.1451 [hep-ph]

40. A.D. Martin, W. Stirling, R. Thorne, G. Watt, Eur. Phys. J. C 63, 189 (2009)

41. R.D. Ball, et al., Nucl. Phys. B 855, 153 (2012). 2012

42. S. Alekhin, J. Blümlein, S. Moch, arXiv:1202.2281 [hep-ph]

43. D.L. Rainwater, D. Zeppenfeld, J. High Energy Phys. 9712, 005 (1997)

44. D. Rainwater, D. Zeppenfeld, K. Hagiwara, Phys. Rev. D 59, 014037 (1998)

45. D. Rainwater, D. Zeppenfeld, Phys. Rev. D 60, 113004 (1999)

46. T. Plehn, D. Rainwater, D. Zeppenfeld, Phys. Rev. D 61, 093005 (2000)

47. N. Kauer, T. Plehn, D. Rainwater, D. Zeppenfeld, Phys. Lett. B 503, 113 (2001)

48. V. del Duca et al., Phys. Rev. Lett. 87, 122001 (2001)

49. V. del Duca et al., Nucl. Phys. B 616, 367 (2001)

50. G. Klamke, D. Zeppenfeld, J. High Energy Phys. 0704, 052 (2007)

51. J. Campbell, R. Ellis, G. Zanderighi, J. High Energy Phys. 10, 028 (2006)

52. J.M. Campbell et al., arXiv: 1202.5475

53. C.F. Berger et al., J. High Energy Phys. 1104, 092 (2011)

54. I.W. Stewart, F.J. Tackmann, Phys. Rev. D 85, 034011 (2012)

55. A. Banfi, G.P. Salam, G. Zanderighi, J. High Energy Phys. 1206, 159 (2012)

56. S. Dittmaier, et al. (LHC Higgs XS WG). arXiv:1201.3084 [hep$\mathrm{ph}]$ 
57. D. Zeppenfeld, R. Kinnunen, A. Nikitenko, E. Richter-Was, Phys. Rev. D 62, 013009 (2000)

58. A. Djouadi et al., hep-ph/0002258

59. M. Dührssen et al., Phys. Rev. D 70, 113009 (2004)

60. K. Assamagan et al., hep-ph/0406152

61. A. Denner et al., Eur. Phys. J. C 71, 1753 (2011)

62. M. Mangano, J. Rojo, J. High Energy Phys. 1208, 010 (2012)

63. A. Djouadi, J. Kalinowski, M. Spira, Comput. Phys. Commun. 108, 56 (1998)

64. K. Nakamura et al., J. Phys. G 37, 075021 (2010)

65. M. Veltman, Nucl. Phys. B 123, 89 (1977)

66. M. Peskin, T. Takeuchi, Phys. Rev. Lett. 65, 964 (1990)

67. G. Altarelli, R. Barbieri, Phys. Lett. B 253, 161 (1991)

68. M. Farina, C. Grojean, E. Salvioni, J. High Energy Phys. 1207, $012(2012)$

69. B. Mellado, W. Quayle, S.L. Wu, Phys. Lett. B 611, 60 (2005)
70. A. Djouadi, A. Falkowski, Y. Mambrini, J. Quevillon, arXiv:1205. 3169 [hep-ph]

71. J. Butterworth, A. Davison, M. Rubin, G. Salam, Phys. Rev. Lett. 100, 242001 (2008)

72. B.A. Kniehl, Phys. Rev. D 42, 2253 (1990)

73. D.A. Dicus, C. Kao, Phys. Rev. D 38, 1008 (1988)

74. O. Brein, A. Djouadi, R. Harlander, Phys. Lett. B 579, 149 (2004)

75. C. Boddy, S. Farrington, C. Hays, arXiv:1208.0769 [hep-ph]

76. A. Djouadi, W. Kilian, M. Muhlleitner, P. Zerwas, Eur. Phys. J. C 10, 45 (1999)

77. S. Dawson, S. Dittmaier, M. Spira, Phys. Rev. D 58, 115012 (1998)

78. ATLAS Collaboration, ATLAS-CONF-2012-091

79. CMS Collaboration, CMS PAS HIG-12-015

80. S. Weinberg, Phys. Rev. Lett. 19, 1264 (1967) 